\title{
A Monoclonal Antibody that Distinguishes between Temporal and Nasal Retinal Axons
}

\author{
Steven C. McLoon \\ Department of Cell Biology and Neuroanatomy, University of Minnesota, Minneapolis, Minnesota 55455
}

\begin{abstract}
A monoclonal antibody was developed that recognizes an antigen with an asymmetric distribution in the chick retina. Immunohistochemistry showed that this antigen, temporal retinal axon protein (TRAP), was present on most if not all axons that arose from the temporal side of the retina. Very few of the axons from the nasal side of the retina were positive for TRAP. The nasal-temporal difference appeared to be in the number of axons that stained with this antibody rather than in the intensity of staining. The transition between nasal and temporal retina based on TRAP distribution appeared to be a vertical line centered on the optic fissure. A competition-based ELISA was developed to quantify the average amount of TRAP on axons in different regions of the retina. This assay also suggested that the pattern of TRAP distribution across the retina was a step function, though the results did not completely rule out the possibility of a continuous concentration gradient oriented circumferentially around the retina. Explants of embryonic nasal and temporal retina had a similar dichotomy in TRAP expression during the first 1 or $2 d$ in culture. The antibody to TRAP bound to retinal neurites in culture without the cell membrane being made permeable, which suggests that TRAP is a cell-surface molecule. In culture, TRAP was also expressed on the growth cones. Immunoblots showed that TRAP is trypsin sensitive and has an approximate molecular mass of $135 \mathrm{kDa}$. This is the first molecule identified with an asymmetric distribution in the nasal-temporal axis of the retina. Its dlstribution correlates with previous studies that demonstrated differences in the activity of axons in culture from the nasal and temporal sides of the retina. It is possible that TRAP has a role in development of a topographic pattern of axonal connections between the eye and central visual nuclei in the brain.
\end{abstract}

The axons of retinal ganglion cells project to the central visual nuclei in the brain in a topographic pattern, such that the 2-D pattern of the ganglion cells across the retina is approximately recreated in the pattern of their terminals in the visual nuclei (Hamdi and Whitteridge, 1954). Alteration in the projection pattern of the ganglion cell axons results in ineffective processing of visual information and an inability to respond to visual stim-

\footnotetext{
Received July 11, 1990; revised Nov. 20, 1990; accepted Dec. 21, 1990.

This work was supported by NIH Grant EYO5371 and by the Graduate School of the University of Minnesota.

Correspondence should be addressed to Steven C. McLoon, Department of Cell Biology, University of Minnesota, 4-135 Jackson Hall, 321 Church Street Southeast, Minneapolis, MN 55455.

Copyright (C) 1991 Society for Neuroscience $0270-6474 / 91 / 111470-08 \$ 03.00 / 0$
}

uli in a meaningful manner (So et al., 1981). The mechanism responsible for development of this orderly pattern of connections is unknown. One theory that has gained wide acceptance and has considerable experimental support is the chcmoaffinity theory (Sperry, 1963). This theory proposes that neurons acquire positionally dependent chemical labels and that the pattern of connections is due to the selective affinity between the labels carried on the growing axons and the cells to which they connect. The genome seems too small to allow for a unique chemical label on each ganglion cell. Alternatively, two molecules distributed across the retina in concentration gradients perpendicular to one another could impart positional labels to these cells (Fraser, 1980; Gierer, 1983).

A number of studies have demonstrated differences between cells in dorsal and ventral retina that may reflect positional labels in this axis. These studies described differences in the ability of dorsal and ventral retinal cells to interact both with each other (e.g., Gottlieb et al., 1976) and with cells in different regions of the optic tectum (e.g., Barbera, 1975). Several molecules have becn identified in dorsal-ventral concentration gradients in the developing retina (Marchase, 1977; Trisler et al., 1981; Irwin et al., 1985; Constantine-Paton et al., 1986; Rabacchi et al., 1990). However, there is still no definitive evidence that shows these molecules function as positional labels for growing axons.

A dorsal-ventral gradient would give positional information for one dimension of the 2-D retinal map. For each cell in the map to have a unique positional label, at least one more positional vector would be required, possibly a nasal-temporal gradient. Although early studies using cell membranes failed to identify nasal-temporal differences in the retina (e.g., Barbera, 1975; Gottlieb et al., 1976), more recent studies, which used live retinal axons, have demonstrated such differences (e.g., Halfter et al., 1981; Bonhoeffer and Huf, 1982). However, no molecules have previously been reported as having a nasaltemporal concentration difference in the retina.

This study describes a molecule, identified by virtue of its binding by a monoclonal antibody, with an asymmetrical distribution in the nasal-temporal axis of the retina.

\section{Materials and Methods}

Animals. Newborn mice were obtained for the production of monoclonal antibodies by mating 6-7-week-old $\mathrm{Balb} / \mathrm{c}$ mice (from HarlanSprague Dawley Inc.).

Fertilized chicken eggs, pathogen-free White Leghorn crossed with Rhode Island Red, were obtained from the University of Minnesota Poultry Research Center. Eggs were incubated at $37^{\circ} \mathrm{C}$ in an egg incubator. Some embryos were removed from the shell after $3 \mathrm{~d}$ of incubation and transferred to culture chambers (McLoon, 1985). Cultured embryos had both eye vesicles destroyed by microcautery on the day they were removed from the shells. The eyeless embryos were maintained in a 
forced-draft culture incubator at $37^{\circ} \mathrm{C}$, with $95 \%$ relative humidity and $1 \% \mathrm{CO}_{2}$.

Monoclonal antibody production. Monoclonal antibodies were prepared by a subtractive immunization protocol (Hockfield, 1987; Vermeersch et al., 1991). The lower half of tecta were dissected from eyeless embryos on day 9 of incubation (E9) and homogenized in a small amount of phosphate-buffered salinc. The total protein concentration in the tissue homogenate was determined in an aliquot of the sample solubilized in $0.5 \%$ Triton X-100 using the BCA assay (Pierce). The homogenate was diluted to $5 \mathrm{mg}$ protein $/ \mathrm{ml}$. A litter of mice within $12 \mathrm{hr}$ of birth received an intraperitoneal injection of $20 \mu \mathrm{l}$ diluted tissue homogenate. After 3 weeks, these mice were injected with a homogenate of tectum prepared as described above from normal chick embryos; 100 $\mu \mathrm{l}$ of the homogenate was injected into the tail vein, and $400 \mu \mathrm{l}$ of the homogenate mixed 1:1 with Freund's complete adjuvant was injected intraperitoneally. This was repeated 2 weeks later, again with normal chick tectum. Three days after the booster, the spleens were removed from two of the mice. Hybridomas were produced by fusion of the spleen cells with NS-1 myeloma cells according to standard protocols (Kohler and Milstein, 1975). Hybridoma culture supernatants were screened by immunohistochemistry on frozen scctions of paraformaldehyde-fixed E9 chick retina as described below. One antibody, RA5, was selected for further analysis based on its selective binding to axons from the temporal side of the retina. The RA5-producing colony was subcloned twice by limiting dilution and then expanded in $75-\mathrm{cm}^{2}$ flasks. Culture supernatant was harvested and centrifuged at $1500 \times g$ for 10 min. The supernatant from this low-speed spin was used as the "RA5 antibody" in all the following procedures.

Immunohistochemistry. Immunohistochemistry was used to study the distribution of RA5 immunoreactivity in retinas of different ages. Embryos from E3 to E5 were fixed by immersion in 4\% paraformaldehyde/ $0.1 \mathrm{M}$ phosphate buffer. Chicks, E6 and older, were perfused through the heart with the same fixative. Eyes and brains of the chicks were cryoprotected in $20 \%$ sucrose/phosphate buffer overnight, embedded in a homogenate of brain, and sectioned at $12 \mu \mathrm{m}$ in a cryostat. The scctions were mounted on chrome-alum-coated glass slides. After rinsing the slides in PBS, nonspecific staining was blocked by incubation of the sections in $10 \%$ normal goat serum/PBS. The sections were then incubated for $1.5 \mathrm{hr}$ in the RA5 antibody, rinsed in PBS, and incubated for $1 \mathrm{hr}$ in goat anti-mouse IgG antibody conjugated to fluorescein isothiocyanate (Cappel). The slides were examined and photographed with an epifluorescence microscope.

Adjacent sections were processed as controls. These were processed as described above except that they were incubated in fresh culture medium or a monoclonal antibody that does not bind chick retina in place of the RA5 antibody. No significant fluorescence was detected in the control slides.

Retinal explant cultures. Retinas were dissected from E4 chicks, and small squares were cut from the most peripheral portions of the nasal or temporal sides of the retinas. The retinal squares were cultured on laminin-coated coverslips in defined medium (McLoon and Barnes, 1989). The explants were processed for immunohistochemistry after 1 , 2,4 , or $6 \mathrm{~d}$ in culture. The medium was changed to $\mathrm{F} 12$ with $12 \%$ horse serum and $3 \%$ fetal calf serum. A volume of the RA5 antibody equal to that of the medium in the dish was added to each live culture for 1 hr. The cultures were then rinsed in two changes of F12 medium over a 10-min period and fixed with $2 \%$ paraformaldehyde, $0.5 \%$ dextrose, and $0.1 \mathrm{M}$ phosphate buffer $(\mathrm{pH}, 7.3)$ for $1 \mathrm{hr}$ at $4^{\circ} \mathrm{C}$. The cultures were next rinsed in PBS and treated with a secondary antibody as described above. The coverslips with the explants were inverted onto a microscope slide and examined by fluorescence microscopy.

Immunoblots. The relative molecular weight of TRAP was determined by means of immunoblots. Retinas were dissected from E8-9 chick embryos and homogenized in an SDS sample buffer. The homogenate was centrifuged at $17,000 \times g$ for $10 \mathrm{~min}$, and the supernatant was electrophoresed through a $5 \%$ polyacrylamide gel (Laemmli, 1970). The proteins were transferred from the gel to nitrocellulose electrophoretically (Towbin et al., 1979). Strips of the blots were blocked with $10 \%$ BSA/PBS, incubated for $2 \mathrm{hr}$ in the RA5 antibody, rinsed in PBS, and incubated with goat anti-mouse IgG antibodies conjugated to HRP (Sigma) for $2 \mathrm{hr}$. After rinsing in PBS, the strips were incubated in diaminobenzidine and hydrogen peroxide. Control strips were treated in an identical fashion, except culture medium from a hybridoma cell line not producing any detectable antibodies was used in place of the RA5 antibody. In some cases the tissue was homogenized and incubated in $0.05 \%$ trypsin in a calcium/magnesium-free Hank's medium for 15 min at $37^{\circ} \mathrm{C}$ prior to homogenization in the sample buffer. Trypsintreated samples were processed as described above. Protein standards ranging in molecular weight from 84 to $205 \mathrm{kDa}$ were run in an additional lane of the gel and visualized on the blot with Aurodye (Janssen).

$C$-ELISAs. A competition-based enzyme-linked immunosorbent assay (C-ELISA) was used to determine the relative amount of TRAP and $\mathrm{L} 1$ in different regions of the retina. Unlike a standard ELISA, which is most effective for showing relative amounts of antibody, the C-ELISA shows a linear relationship between antigen concentration and optical density (OD). The assay used either a known or an unknown amount of soluble antigen mixed with the antibody to alter the amount of antibody available to bind a fixed amount of antigen on the plate. The assay in effect measured the amount of antibody that remained after treatment with the test antigen. The assay conditions described here were determined empirically to give the maximum signal-to-noise ratio.

Retinas were dissected in PBS from E9 chick embryos for use in preparing ELISA plates. The tissue was homogenized in $36 \mathrm{~mm} n$-octyl $\beta$ - $d$-glucopyranoside, $70 \mu \mathrm{g} / \mathrm{ml}$ aprotinin, $100 \mu \mathrm{g} / \mathrm{ml}$ leupeptin, $30 \mu \mathrm{g} /$ $\mathrm{ml}$ pepstatin A, $1 \mu \mathrm{g} / \mathrm{ml}$ phenyl methyl-sulfonyl fluoride (PMSF), and PBS on ice and centrifuged for $10 \mathrm{~min}$ at $17,000 \times \mathrm{g}$. The supernatant was dialyzed against PBS, after which the protein concentration in the sample was determined by a BCA assay (Pierce). Some of the sample at this point was reserved for generating a calibration curve. The remaining sample was diluted to $200 \mu \mathrm{g} / \mathrm{ml}$ in $15 \mathrm{mM} \mathrm{Na}_{2} \mathrm{CO}_{3}$ and 35 mM NaHCO $(\mathrm{pH}, 9)$. Each well of NUNC ELISA plates received 50 $\mu \mathrm{l}$ of this diluted sample. The plates were centrifuged for $10 \mathrm{~min}$ at 1500 $\times g$ at $4^{\circ} \mathrm{C}$ and dried under forced air at $37^{\circ} \mathrm{C}$. Plates were stored at $-75^{\circ} \mathrm{C}$ until use.

The optimal concentration of both primary antibodies were determined by running a dilution series with a standard ELISA. All incubations of the plates were done at room temperature on a rotating platform at $80 \mathrm{rpm}$. Nonspecific binding was blocked by treating each well with $2 \%$ horse serum and PBS for $60 \mathrm{~min}$. Then, each well was incubated with diluted primary antibody, either RA5 or 8D9, an antibody to chick L1 (Lemmon and McLoon, 1986). Serial dilutions of each antibody between $10^{-1}$ and $10^{-8}$ were tested. After a 1-hr incubation in primary antibody, the wells were rinsed in PBS with a plate washer. The wells were incubated $30 \mathrm{~min}$ in biotinylated anti-mouse IgG secondary antibody (Vector) diluted 1:2000. After further rinsing, the wells were incubated $30 \mathrm{~min}$ in avidin-HRP complex (Elite Vectastain Kit, Vector, with components A and B each at $1: 1000$ in $0.1 \%$ Tween-20 and PBS). The wells were again rinsed in PBS and then incubated in an $o$-phenyldiamine (OPD) substrate ( $2 \mathrm{mM}$ OPD, $0.01 \% \mathrm{H}_{2} \mathrm{O}_{2}, 20 \mathrm{~mm}$ citric acid, and $50 \mathrm{~mm}$ sodium phosphate at $\mathrm{pH} 5.3$ ). The reaction was stopped in each well after sufficient development by the addition of $1 \mathrm{~N}$ $\mathrm{H}_{2} \mathrm{SO}_{4}$. The optical density of each well was read at $492 \mathrm{~nm}$. A dilution curve was plotted. The antibody dilution corresponding to the upper shoulder of the curve was selected for all further experiments. This was a 1:100 dilution for 8D9 and a 1:300 dilution for RA5.

Retinas from E9 chick embryos were cut into 18 segments as diagrammed in Figure 1 for preparation of the competition protein. Similar segments from each of 20-24 eyes were pooled. Each sample was homogenized, extracted, and dialyzed as described above. The total protein concentration in each sample was determined by a micro-BCA assay (Pierce). An aliquot of each sample was diluted in aliquots of primary antibody. Two sets of antibody-sample mixtures were prepared, one with the competition protein at $200 \mu \mathrm{g} / \mathrm{ml}$ and the other at $20 \mu \mathrm{g} / \mathrm{ml}$ (referred to as $1 \times$ or $10 \times$ dilution, respectively, in Table 1). The antibody-sample mixtures were used as the primary antibody to run an assay as described above. Each sample was run in a set of four duplicate wells. Different amounts of sample prepared from total retina were mixed with the primary antibody and run in parallel with the samples of individual retinal segments. These results were used to prepare a calibration curve, which was approximately linear over the range of the test (Fig. 2). Regression analysis was used to convert the optical density data for each retinal segment into a relative amount of antigen per sample based on the calibration curve. This gave the relative amounts of TRAP and $L 1$ in each retinal segment.

\section{Results}

In order to produce monoclonal antibodies to rare antigens of developing retinal axons, mice within hours of birth were injected with brain from eyeless chick embryos. The purpose of 


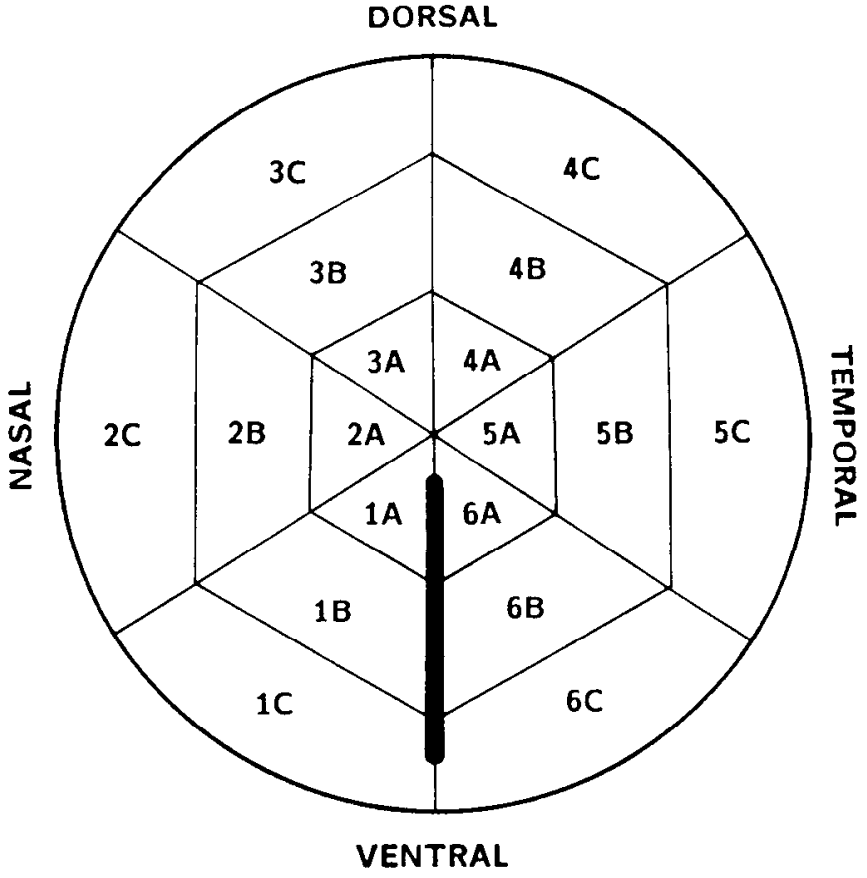

Figure 1. Diagram of a chick retina showing the pattern in which the retinas were cut for the regional assay. The optic fissure is represented by the dark bar in the lower center.

this injection was to make mice immunologically tolerant to common antigens of the developing brain. These mice were later immunized with tecta from normal chick embryos, and finally, antibody-producing hybridomas were generated from the spleens of these mice. A number of monoclonal antibodies that recognize developing retinal axons were obtained from these hybridomas. One of these, RA5, appeared to recognize a protein present on most if not all axons from temporal retina and was virtually absent from nasal retinal axons. The working name for this protein is TRAP (temporal retinal axon protein). The following is an initial characterization of TRAP as identified by the monoclonal antibody in the developing chick retina.

\section{Immunohistochemistry}

Eyes from chicks of various ages from embryos on E3 to adult ( 2 months after hatching) were processed for fluorescence immunohistochemistry using the antibody to TRAP. Late on E3, axons in the newly developed optic fiber layer of the retina just dorsal to the optic stalk were positive for TRAP. The staining was a small patch, not clearly nasal or temporal in position (not shown). From E6 through adult, most if not all axons in temporal retina stained for TRAP, while very few of the axons in nasal retina stained (Fig. 3). The nasal-temporal difference appeared to be in the number of axons that stained rather than a graded intensity of staining. The transition between nasal and temporal retinal staining pattern for TRAP in the optic fiber layer was rather abrupt. In the ventral half of the retina, this transition was centered on the optic fissure. If the optic fissure were to be extended as a straight line into the dorsal half of the retina, it would mark the transition in this region, as well. Control sections treated with a monoclonal antibody that does not bind to antigens in chick retina showed no staining. Also, sections stained with antibodies to other molecules associated with embryonic chick retinal axons (i.e., L1, RA4, Thy-1, N-CAM)

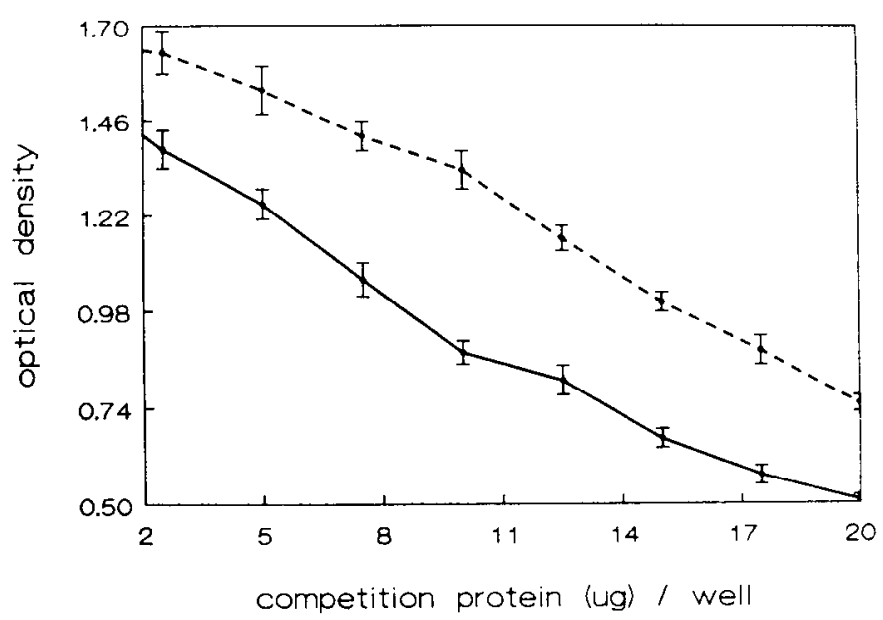

Figure 2. Calibration curve of the C-ELISA showing the assay response (OD) in relation to the amount of retinal extract ( $\mu \mathrm{g}$ competition protein) added to the RA5 (solid line) or 8D9 (broken line) primary antibody. Error bars indicate SD.

showed no nasal-temporal differences in their staining patterns (data not shown).

As carly as E8 in central retina and somewhat later in more peripheral retina, the antibody also stained a lamina of fibers in the inner plexiform layer. This lamina was immediately adjacent to the inner nuclear layer. Stained fibers could occasionally be traced from this lamina to the optic fiber layer. There was no obvious nasal-temporal difference in the staining of this lamina.

\section{Retinal explant cultures}

Immunohistochemical analysis of retinal explant cultures was used to determine the subcellular location of TRAP and whether the asymmetry in TRAP expression was maintained in culture. Explants of nasal or temporal E4 retina were cultured for $1 \mathrm{~d}$ on a laminin substrate and then stained live by immunohistochemistry with the antibody to TRAP (Fig. 4). No staining was observed on neurites from nasal explants. All neurites from the temporal explants were TRAP positive. Staining was present along the entire length of the neurites, including the growth cones and their filopodia and lamellipodia. Control cultures processed in the same manner with a primary antibody to chick neurofilaments, an intracellular antigen, showed no staining. This suggests that the TRAP is on the cell surface, a characteristic that would be expected if this molecule were to function in some type of cell-cell or cell-matrix recognition.

After $2 \mathrm{~d}$ in culture, $42 \%$ of the nasal retinal explants had some TRAP-positive neurites. Because the explants were taken from very young peripheral retina, the neurites that grow out in culture represent new axon growth rather than regenerating axons. It may be that, for a given nasal region of retina in vivo, the first axons to grow out are TRAP negative, and axons that grow out later from that portion of retina are TRAP positive. This might explain the late appearance of TRAP-positive neurites in cultures of nasal explants.

Cultures that were maintained for 4 or $6 \mathrm{~d}$ showed a reduction in staining intensity on the neurites from explants of temporal retina, and some neurites from all explants of nasal retina expressed TRAP. By $8 \mathrm{~d}$ in culture, the neurites appeared unhealthy, and there was no definitive staining for TRAP with nasal or temporal explants. 

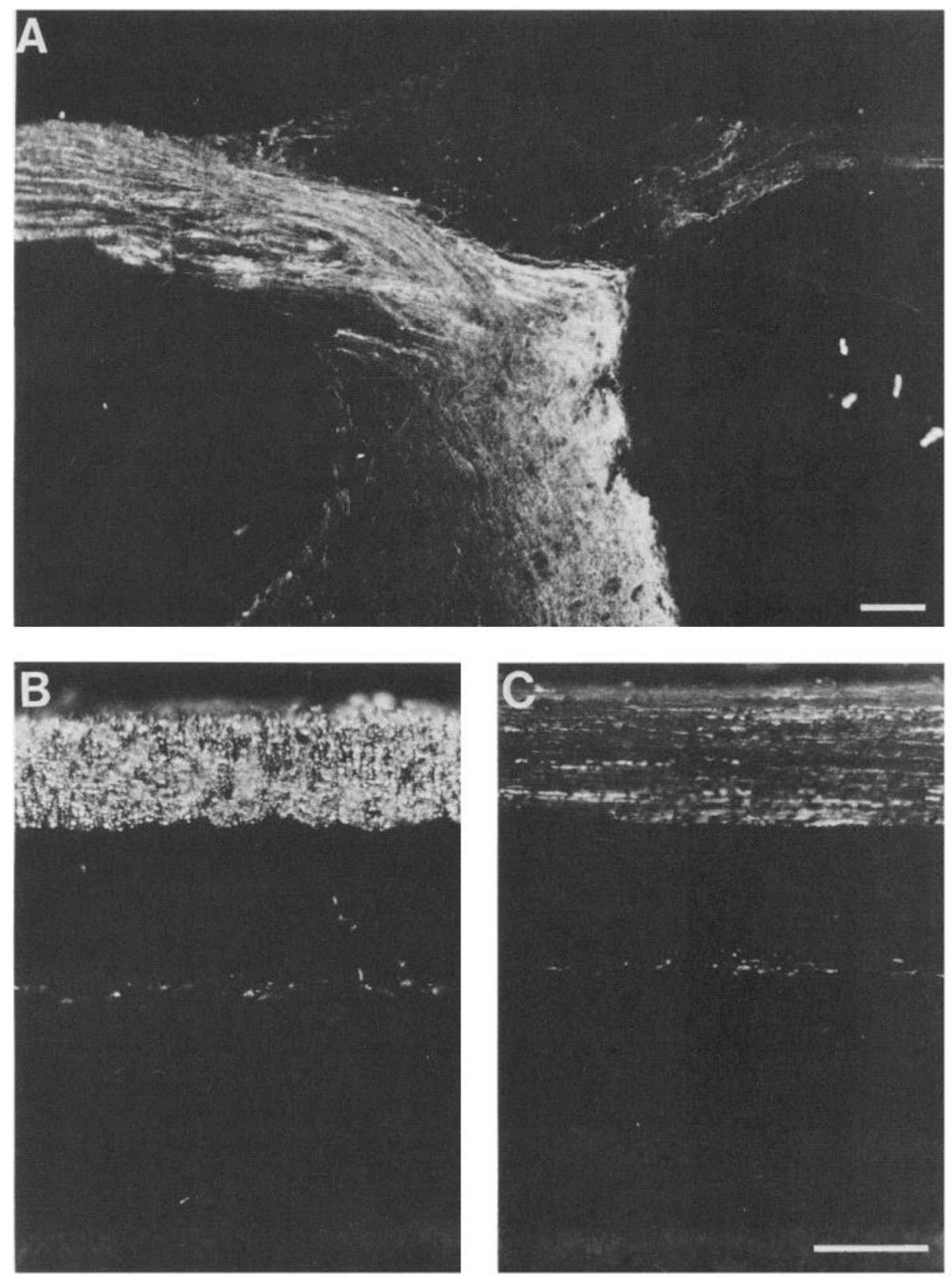

Figure 3. Fluorescence photomicrograph of sections of chick retina stained immunohistochemically with the RA5 antibody. The optic fiber layer is towards the top. $A$, E10 retina through the optic nerve. Temporal retina is toward the left, nasal retina is toward the right, and the optic nerve is oriented vertically in the center. Most, if not all, axons on the temporal side are RA5 positive, and very few of the axons on the nasal side are RA5 positive. RA5positive axons run from the optic fiber layer into the optic nerve. $B$ and $C$, Temporal and nasal side, respectively, of the same section of an adult retina. The thick band of positive staining is in the optic fiber layer. The thin band of positive staining in the center is in the inner plexiform layer adjacent to the inner nuclear layer. Scale bars, 50 $\mu \mathrm{m}$.

\section{Immunoblots}

Initial characterization of the TRAP antigen suggests that it is a protein or glycoprotein with a molecular weight of 135,000 $\mathrm{Da}$. An SDS extract of E8-9 retinas was electrophoresed on a $5 \%$ polyacrylamide gel. Proteins in the gel were transferred to nitrocellulose by electrophoresis and stained with the TRAP antibody. This revealed a single tightly focused band with a molecular weight of $\sim 135,000 \mathrm{Da}$ (Fig. 5, lane A). Antibody binding was lost when the extract was treated with trypsin prior to electrophoresis, which is indicative of the antigen being a protein (Fig. 5, lane B). No bands were detected in a blot reacted with a control monoclonal antibody (Fig. 5, lane C).

\section{C-ELISA}

The interest in TRAP lies in the obvious possibility that it may serve as a positional marker on the axons of the developing retina. In order to obtain a better indication of the possible role of TRAP, it seemed necessary to quantify the concentration of TRAP on axons in different regions of the retina. Because TRAP appears to be present mainly on axons and because the number of axons varies from region to region across the retina, quantitation of TRAP in different regions of the retina would not reveal the concentration of TRAP on individual axons. Instead, the $\mathrm{L} 1$ antigen, identified with the 8D9 antibody, was quantified in different regions of the retina as an indication of the relative amount of ganglion cell axons in the different regions. $\mathrm{Ll}$ is a cell adhesion molecule that in the developing chick retina is restricted to the optic axons, and it appears to be evenly distributed on the axons across the retina (Lemmon and McLoon, 1986). The relative concentration of TRAP in each region of the retina was also determined. A ratio of the relative amount of TRAP to the relative amount of $\mathrm{Ll}$ was used as an indication of the average concentration of TRAP per axon in the different regions of the retina. This ratio was determined for 18 segments of the retina (Fig. 6, Table 1). The retina was divided into six 

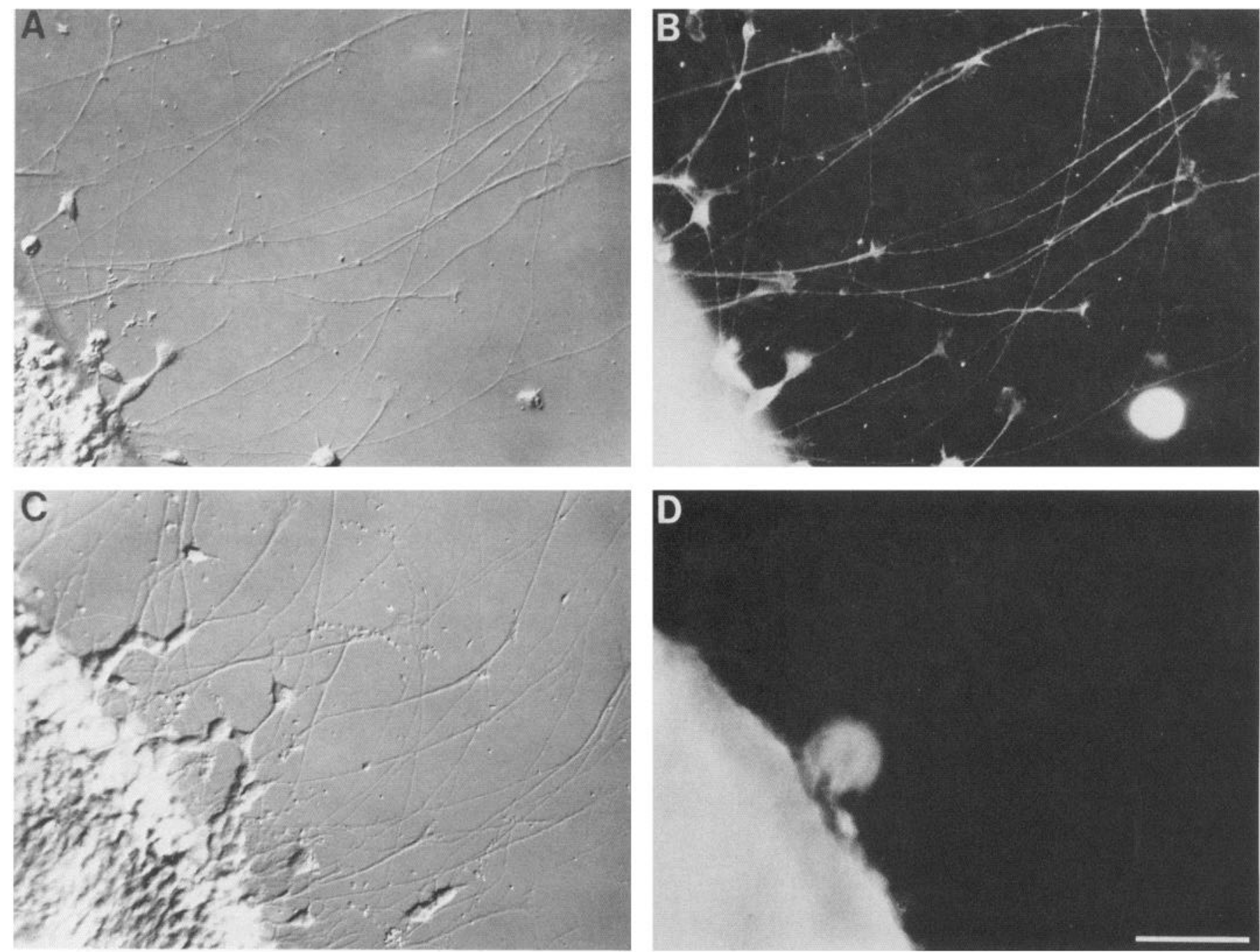

Figure 4. Retinal explants after $1 \mathrm{~d}$ in culture stained live with the RA5 antibody. They are photographed with differential interference microscopy to show all processes (left) and fluorescence microscopy to show labeling with the antibody (right). The top explant $(A, B)$ was derived from the temporal side of a retina. The neurites, including the growth cones, are RA5 positive. The lower explant $(C, D)$ was derived from the nasal side of a retina, and none of the neurites are RA5 positive. Scale bar, $50 \mu \mathrm{m}$.

wedges, much like a pie would be cut. Each wedge was cut into three segments representing central, intermediate, and peripheral retina. There was no significant central-peripheral difference in the amount of TRAP per axon, as indicated by the TRAP: L1 ratio, in any single segment. However, there was a large difference between segments. The TRAP ratio was virtually the same for the lower and middle temporal segments and also for lower and middle nasal segments. The lower temporal segments had approximately 30 times more TRAP per axon than the lower nasal segments. The upper temporal and nasal segments had values intermediate between each of their adjacent segments. This assay was run three times with similar results.

\section{Discussion}

In the process of generating monoclonal antibodies to developing chick retinal axons, one antibody was identified that appeared to recognize an antigen that is much more abundant on the temporal side of the retina than on the nasal side. The working name for this antigen is TRAP (temporal retinal axon protein).
In the optic fiber layer, TRAP is associated with the retinal axons. The immunohistochemical staining pattern in sectioned retinas is consistent with this conclusion. TRAP immunoreactivity was also present on the neurites and growth cones that extended from explants of developing temporal retina. The processes from retinal explants arise strictly from the retinal ganglion cells (Johns et al., 1978; Halfter et al., 1983). TRAP immunoreactivity in sectioned retinas was associated with most if not all axons on the temporal side of the retina, and it appeared to be present on only a small percentage of the axons on the nasal side. The axons that arose from explants of the nasal or temporal side of developing retina revealed a similar dichotomy in TRAP expression after $1 \mathrm{~d}$ in culture.

TRAP-positive processes were also present to a limited extent in the inner plexiform layer. These processes could occasionally be seen to join the optic fiber layer; thus, it is possible that this staining was on processes of the displaced ganglion cells. A layer of displaced ganglion cells is known to be present along the inner border of the inner nuclear layer (Karten et al., 1977). Because the staining pattern in the inner plexiform layer was fairly uni- 
A

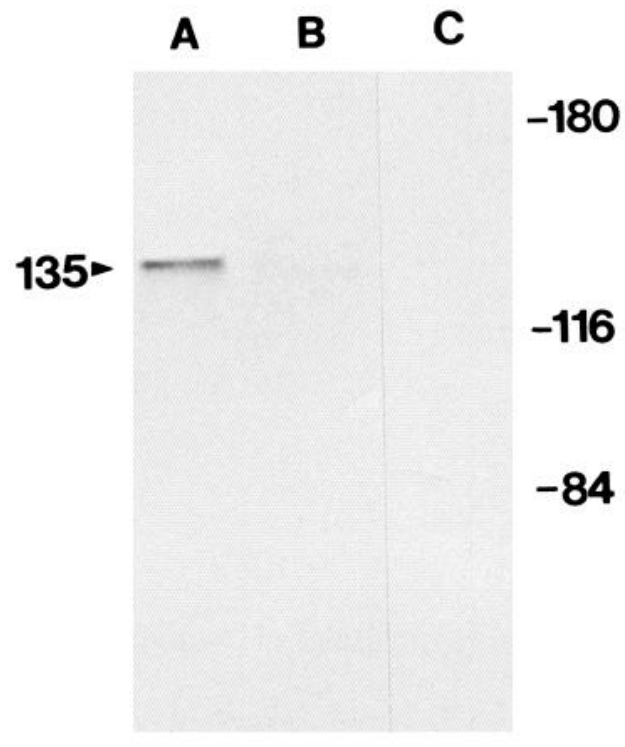

Figure 5. Immunoblots of extract from E8-9 chick retinas. Lane A was untreated extract reacted with the RA5 antibody. It shows a single tightly focused band at approximately $135 \mathrm{kDa}$. Lane $B$ was a trypsintreated extract reacted with the RA5 antibody. Immunoreactivity was mostly abolished. Lane $C$ was untreated extract reacted with a control culture supernatant. No immunoreactivity was visible. Molecular weight indicators along the right side are in kilodaltons.

form across the entire retina, it may be that displaced ganglion cells do not have a nasal-temporal difference in TRAP expression. To extend this possibility further, the few TRAP-positive axons in the optic fiber layer on the nasal side of the retina could arise from displaced ganglion cells.

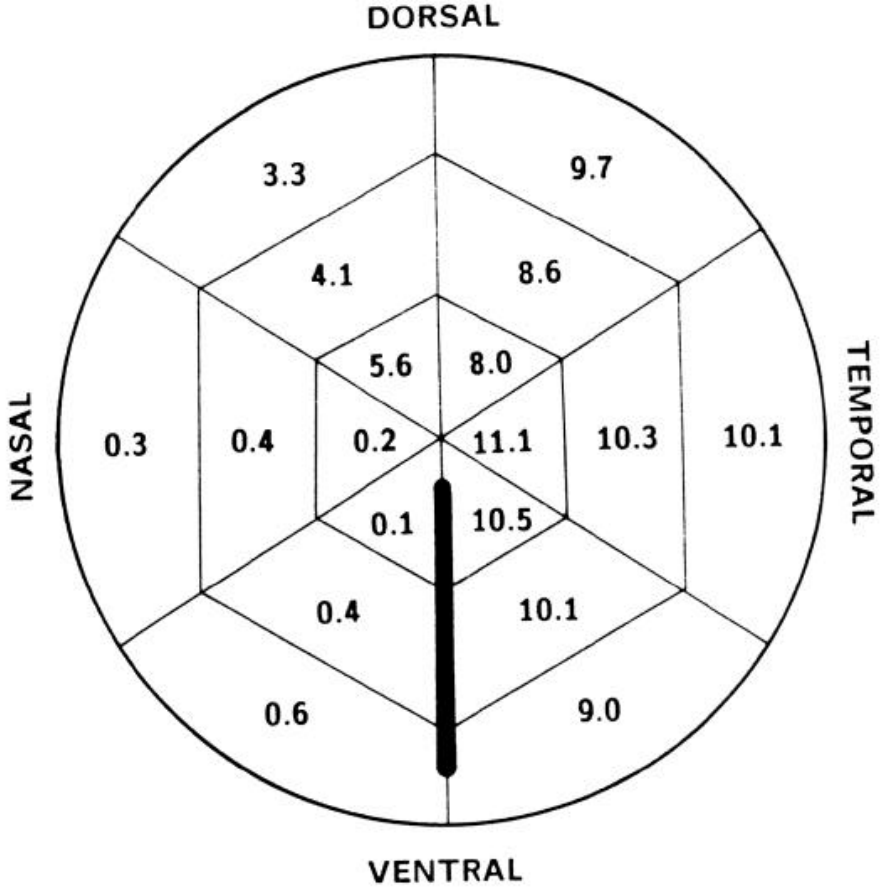

Figure 6. Diagram as in Figure 1 of E9 chick retina showing the ratio of TRAP to $\mathrm{Ll}$ in different regions.

The immunohistochemistry suggested that TRAP distribution was a step function from one side of the retina to the other, rather than a continuous concentration gradient. However, as has been shown for other molecules in the retina, immunohistochemistry is not always sufficiently quantitative to reveal a gra-

Table 1. Assay of TRAP and L1 in different retinal segments by C-ELISA

\begin{tabular}{|c|c|c|c|c|c|}
\hline \multirow{2}{*}{$\begin{array}{l}\text { Retinal } \\
\text { segment }\end{array}$} & \multicolumn{2}{|l|}{ OD (dilution) } & \multicolumn{2}{|c|}{$\begin{array}{l}\text { Relative antigen } \\
\text { concentration }\end{array}$} & \multirow{2}{*}{$\begin{array}{l}\text { TRAP:L1 } \\
\text { ratio }\end{array}$} \\
\hline & TRAP & $\mathrm{Ll}$ & TRAP & $\mathrm{L} 1$ & \\
\hline $1 \mathrm{~A}$ & $1.34 \pm 0.08$ & $0.92 \pm 0.02(1)$ & 2.4 & 17.0 & 0.1 \\
\hline 1B & $1.25 \pm 0.04(1)$ & $1.28 \pm 0.03(1)$ & 4.3 & 10.0 & 0.4 \\
\hline $1 \mathrm{C}$ & $1.14 \pm 0.02(1)$ & $1.30 \pm 0.04(1)$ & 6.4 & 9.8 & 0.6 \\
\hline $2 \mathrm{~A}$ & $1.31 \pm 0.03(1)$ & $0.96 \pm 0.04$ & 3.1 & 16.1 & 0.2 \\
\hline 2B & $1.17 \pm 0.02(1)$ & $1.03 \pm 0.02(1)$ & 5.8 & 14.7 & 0.4 \\
\hline $2 \mathrm{C}$ & $1.41 \pm 0.08$ & $1.54 \pm 0.05$ & 1.3 & 5.1 & 0.3 \\
\hline $3 A$ & $1.11 \pm 0.03(10)$ & $1.15 \pm 0.01$ & 70.0 & 12.4 & 5.6 \\
\hline $3 B$ & $1.28 \pm 0.05(10)$ & $1.31 \pm 0.03(1)$ & 38.1 & 9.4 & 4.1 \\
\hline $3 \mathrm{C}$ & $0.63 \pm 0.02(1)$ & $1.54 \pm 0.05$ & 16.2 & 5.0 & 3.3 \\
\hline $4 \mathrm{~A}$ & $1.13 \pm 0.02(10)$ & $1.37 \pm 0.04(1)$ & 65.9 & 8.2 & 8.0 \\
\hline 4B & $1.16 \pm 0.05(10)$ & $1.44 \pm 0.05(1)$ & 60.0 & 7.0 & 8.6 \\
\hline $4 \mathrm{C}$ & $1.30 \pm 0.04(10)$ & $1.61 \pm 0.08$ & 35.1 & 3.6 & 9.7 \\
\hline $5 \mathrm{~A}$ & $0.82 \pm 0.02(10)$ & $1.21 \pm 0.02(1)$ & 125.9 & 11.4 & 11.1 \\
\hline $5 B$ & $0.95 \pm 0.03(10)$ & $1.29 \pm 0.03(1)$ & 101.0 & 9.8 & 10.3 \\
\hline $5 \mathrm{C}$ & $1.30 \pm 0.05(10)$ & $1.63 \pm 0.07(1)$ & 33.6 & 3.3 & 10.1 \\
\hline $6 \mathrm{~A}$ & $0.85 \pm 0.02(10)$ & $1.20 \pm 0.03(1)$ & 120.5 & 11.4 & 10.5 \\
\hline $6 \mathrm{~B}$ & $1.16 \pm 0.03(10)$ & $1.49 \pm 0.03(1)$ & 60.8 & 6.0 & 10.1 \\
\hline $6 \mathrm{C}$ & $1.17 \pm 0.02(10)$ & $1.46 \pm 0.06(1)$ & 59.3 & 6.6 & 9.0 \\
\hline
\end{tabular}

Retinal segments are as indicated in Figure 1. OD values are each the mean spectrophotometric reading for four wells \pm SD. A 10 -fold dilution of some of the test samples was required for readings to be in the linear portion of the calibration curve, which is indicated in parentheses. Linear regression generated an equation for both lines in Figure 2 , and this regression was used to convert the OD values to relative concentrations of antigen. The ratios were derived by dividing the relative concentrations of TRAP by the relative concentrations of L1. Four decimal points were carried through all calculations. 
dient in an antigen's distribution (Trisler et al., 1981; Constantine-Paton et al., 1986). It seems unlikely that TRAP is in a continuous nasal-temporal gradient. However, because there was no obvious difference in the staining intensity of axons within the temporal retina, there was a sharp transition between the nasal and temporal staining patterns, and the few TRAPpositive axons in nasal retina appeared to stain with an intensity equal to those in temporal retina. The ELISA results tend to support this conclusion.

To give a better indication of regional differences in the amount of TRAP per axon, a C-ELISA was developed to quantify antigen. The relative amount of TRAP and L1 in each of 18 segments of retina was determined by this assay. $\mathrm{Ll}$ is a cell adhesion molecule that in the retina is only present on the axons (Lemmon and McLoon, 1986). It was assumed that L1 is in equal concentration on all axons of the retina, which is supported by the immunohistochemistry. If this assumption is true, then the amount of $\mathrm{Ll}$ in a retinal segment is proportional to the axonal surface area in that segment. The ratio of the amount of TRAP to the amount of $\mathrm{Ll}$ in a segment is then an indication of the average amount of TRAP per axon in that segment. The TRAP concentrations determincd in this assay could bc interpreted as support for either of two types of gradient patterns. On initial examination, it appears that TRAP is in a continuous concentration gradient that runs in a circumferential pattern around the retina. The highest concentration was in ventraltemporal retina, the lowest was in ventral-nasal retina, and dorsal retina was intermediate between these. However, on closer inspection, it seems more likely that the TRAP distribution is a step function across the retina, as suggested by the immunohistochemistry. Because the dorsal retina lacks a clear landmark to distinguish nasal from temporal retina, the retinas were divided somewhat arbitrarily in this region when preparing tissue for the assay. Thus, the upper nasal and temporal segments would be contaminated by some tissue from the opposite side. If TRAP were in a nasal-temporal step function, then the inability to precisely separate the two sides should result in a reduction in the apparent TRAP concentration for the upper temporal retina and an increase for the upper nasal retina, which is as observed. Furthermore, if TRAP was in a circumferential gradient, then axons coming from dorsal-temporal retina would run through midtemporal retina on their way to the optic fissure. This should lower the average TRAP concentration in midtemporal retina compared to ventral-temporal. Similarly, axons from upper nasal retina should raise TRAP concentration in middle nasal retina. This was not observed, making it more likely that the TRAP distribution is in a step function.

The ELISA study gave the average concentration of TRAP per axon in a segment of retina. This assay cannot tell whether some axons have high levels of TRAP and others little or none in a given segment. The immunohistochemistry suggests that a few nasal axons have "temporal" levels of TRAP and that the majority of nasal axons have none.

TRAP is the first molecule identified with an asymmetric distribution in the nasal-temporal axis of the retina. Its distribution has an interesting correlation with tissue culture studies that demonstrated nasal-temporal retinal differences. The early studies that failed to show nasal-temporal differences in the retina used dissociated retinal cells or membranes (Barbera, 1975; Gottlieb et al., 1976). The more recent studies that successfully demonstrated nasal-temporal retinal differences used growing retinal axons (Halfter et al., 1981; Bonhoeffer and Huf,
1982, 1985; Walter et al., 1987a). This suggests that nasal-temporal differences in the retina may be confined to the axons, and in the retina, TRAP appears to be restricted to the axons. Tissue culture studies that revealed differences between nasal and temporal retinal axons suggest the existence of a sharp boundary between nasal and temporal characteristics (see I Ialfter et al., 1981, their Fig. 3; Walter et al., 1987b). If TRAP were distributed in a nasal-temporal step function, as appears to be the case, it would also correlate with the activity exhibited in these assays.

Preliminary characterization of TRAP suggests that it is a cell-surface protein or glycoprotein with a molecular mass of approximately $135 \mathrm{kDa}$. The molecular weight was determined by comparing the relative mobility of the antigen with several protein standards in immunoblots. When the tissue homogenate was treated with trypsin prior to electrophoresis, the antibody failed to recognize the antigen in an immunoblot. This suggests that the antigen is a protein or glycoprotein. The antibody bound to live retinal axons and growth cones in tissue culture, which suggests that TRAP is a cell-surface molecule. Antibodies to intracellular proteins failed to stain axons in controls and in similar assays (McLoon and Barnes, 1989).

Expression of TRAP on the surface of retinal axon growth cones is consistent with the possibility that this protein is involved in some type of cell-cell or cell-matrix interaction during axon growth. Its asymmetric distribution on retinal axons makes it tempting to speculate that it has a role in development of patterned neuronal connections. Numerous mechanisms have been suggested to explain how orderly patterns of neuronal connections develop between the eye and central visual nuclei. Many experiments support the chemoaffinity hypothesis, which suggests that neurons in the retina and central visual nuclei independently acquire positional labels, and that the pattern of connections is due to the selective affinity between the labels carried on the growing axons and the cells to which they connect (Sperry, 1963). Positional information is probably encoded by molecules with asymmetric distributions across the developing retina.

There are several possible ways in which asymmetric patterns of molecules could be used to generate unique positional labels on retinal cells. Two possibilities seem most likely. First, two molecules could be distributed on the ganglion cells across the retina in two continuous concentration gradients approximately perpendicular to one another. This would allow for each cell to have a unique label by virtue of the amount of each of the two gradient molecules. Several molecules have been identified that are distributed in a dorsal-ventral concentration gradient in the retina (Marchase, 1977; Trisler et al., 1981; Irwin et al., 1985; Constantine-Paton et al., 1986; Rabacchi et al., 1990). If TRAP were distributed in a circumferential gradient, it would correlate with an anterior-posterior gradient. Prior to closure of the optic fissure, the lower temporal retina is most posterior, and the lower nasal retina is most anterior. TRAP could then supply the second dimension in a two-gradient positional marker system. However, it seems unlikely that TRAP is in a circumferential gradient.

A second possibility is that a dorsal-ventral gradient is combined with a central-peripheral gradient. It is easy to imagine how a central-peripheral gradient could be established during development. Retinal cells are progressively generated and mature in a central-to-peripheral pattern (Kahn, 1974). Some substance progressively lost by serial dilution through division of a stem cell would result in a high-to-low gradient of the sub- 
stance from central to peripheral retina. In the nervous system of $C$. elegans, this type of mechanism appears responsible for the development of positional information (Horvitz, 1981). Also, a progressive increase or decrease in expression of some molecule during maturation of a cell could result in a temporary central-peripheral chemical gradient across the retina during development that reflects the gradient of maturation. Molecules themselves can be expressed in different forms on cells as they mature, which could also result in a maturation-dependent gradient across the retina. A maturational change in the structure of N-CAM has been clearly demonstrated (Pollerberg et al., 1986), and N-CAM is present in the developing retina (Edelman, 1984). However, a central-peripheral gradient on top of the dorsal-ventral gradient would result in a mirror-symmetrical duplication of positional labels on the two sides of the retina. If the central-peripheral gradient was coupled with a unique nasal or temporal retinal marker along with the dorsal-ventral gradient, then this could allow for unique positional labels across the entire retina. Tissue culture studies suggest a very sharp border (rather than a continuous gradient) between nasal and temporal retinal characteristics (e.g., Halfter et al., 1981). It also seems most likely that the TRAP distribution is a step function across the retina and could serve to distinguish temporal from nasal retinal axons.

Several cellular functions could be mediated by TRAP other than selective adhesion between axons and target cells. Tissue culture studies have shown that posterior tectal cells express some factor on their surfaces that inhibits the growth of temporal retinal axons (Walter et al., 1987b; Cox et al., 1990). This factor has virtually no activity on nasal retinal axons. It is possible that TRAP is a receptor for this axon growth inhibitor. These molecules could help prevent temporal retinal axons from inappropriately remaining in posterior tectum (Crossland et al., 1974; McLoon, 1982). Specific adhesive interactions between positionally related retinal axons (rather than between the axons and target cells) may also be important in development of the orderly pattern of axonal projections. In vitro studies have demonstrated that axons from temporal retina grow preferentially on other temporal axons when given a choice between nasal and temporal axons (Bonhoeffer and Huf, 1985). In the embryo, there also appears to be a strong bias toward temporal axons associating with other temporal axons (Fawcett et al., 1984). If TRAP were a homophilically binding cell adhesion molecule, it could function in the fasciculation of temporal retinal axons.

\section{References}

Barbera AJ (1975) Adhesive recognition between developing retinal cells and the optic tecta of the chick embryo. Dev Biol 46:167-191.

Bonhoeffer F, Huf J (1982) In vitro experiments on axon guidance demonstrating an anterior-posterior gradient on the tectum. EMBO J 1:427-431.

Bonhoeffer F, Huf J (1985) Position-dependent properties of retinal axons and their growth cones. Nature 315:409-410.

Constantine-Paton M, Blum AS, Mendez-Otero R, Barnstable C (1986) A cell surface molecule distributed in a dorsoventral gradient in the perinatal rat retina. Nature 324:459-462.

Cox EC, Muller B, Bonhoeffer F (1990) Axonal guidance in the chick visual system: posterior tectal membranes induce collapse of growth cones from the temporal retina. Neuron 2:31-37.

Crossland WJ, Cowan WM, Rogers LA, Kelly JP (1974) The specification of the retino-tectal projection in the chick. J Comp Neurol 155:127-164.

Edelman GM (1984) Cell adhesion molecules. Science 219:450-457.

Fawcett JW, Taylor JSH, Gaze RM, Grant P, Hirst E (1984) Fibre order in the normal Xenopus optic tract, near the chiasma. J Embryol Exp Morphol 83:1-14.
Fraser SE (1980) A differential adhesion approach to the patterning of nerve connections. Dev Biol 79:453-464.

Gierer A (1983) Model for the retino-tectal projection. Proc R Soc Lond [Biol] 218:77-93.

Gottlieb DI, Rock K, Glaser L (1976) A gradient of adhesive specificity in developing avian retina. Proc Natl Acad Sci USA 73:410-414.

Halfter W, Claviez M, Schwarz U (1981) Preferential adhesion of tectal membranes to anterior embryonic chick retina neurites. Nature 292: $67-70$.

Halfter W, Newgreen DF, Sauter J, Schwarz U (1983) Oriented axon outgrowth from avian embryonic retinae in culture. Dev Biol 95:5664.

Hamdi FA, Whitteridge D (1954) The representation of the retina on the optic tectum of the pigeon. Q J Exp Physiol 39:111-119.

Hockfield S (1987) A Mab to unique cerebellar neurons generated by immunosuppression and rapid immunization. Science 237:67-70.

Horvitz HR (1981) Neuronal cell lineages in the nematode Caenorhabditis elegans. In: Development of the nervous system(Garrod D, Feldman J, eds), pp 331-345. Cambridge: Cambridge UP.

Irwin LN, Bremer EG, Irwin CC, McCluer RH (1985) Developmental variation in monosialoganglioside content of embryonic chick retina and tectum. Dev Neurosci 7:239-246.

Johns PR, Heacock AM, Agranoff BW (1978) Neurites in explant culturc of adult goldfish retina derive from ganglion cells. Brain Res 142:531-537.

Kahn AJ (1974) An autoradiographic analysis of the time of appearance of neurons in the developing chick neural retina. Dev Biol 38: $30-40$.

Karten HJ, Fite KV, Brecha N (1977) Specific projection of displaced retinal ganglion cells upon the accessory optic system in the pigeon. Proc Natl Acad Sci USA 74:1753-1756.

Kohler G, Milstein G (1975) Derivation of specific antibody-producing tissue culture and tumor lines by cell infusion. Eur J Immunol 6: 511-519.

Laemmli UK (1970) Cleavage of structural proteins during the assembly of the head of bacteriophage T4. Nature 227:680-685.

Lemmon VP, McLoon SC (1986) The appearance of an L1-like molecule in the chick primary visual pathway. J Ncurosci 6:2987-2994.

Marchase RB (1977) Biochemical studies of retino-tectal specificity. J Cell Biol 75:237-257.

McLoon SC (1982) Alterations in precision of the crossed retinotectal projection during chick development. Science 215:1418-1420.

McLoon SC (1985) Evidence for shifting connections during development of the chick retinotectal projection. J Neurosci 5:2570-2580.

McLoon SC, Barnes RB (1989) Early differentiation of retinal ganglion cells: an axonal protein expressed by premigratory and migrating retinal ganglion cells. J Neurosci 9:1424-1432.

Pollerberg GE, Schachner M, Davoust J (1986) Differentiation statedependent surface mobilities of two forms of the neural cell adhesion molecule. Nature 324:462-465.

Rabacchi SA, Neve RL, Drager UC (1990) A positional marker for the dorsal embryonic retina is homologous to the high-affinity laminin receptor. Development 109:521-531.

So K-F, Schneider GE, Ayres S (1981) Lesions of the brachium of the superior colliculus in neonatal hamsters: correlation of anatomy with behavior. Exp Neurol 72:379-400.

Sperry RW (1963) Chemoaffinity in the orderly growth of nerve fiber patterns and connections. Proc Natl Acad Sci USA 50:703-710.

Towbin H, Staehelin T, Gordon J (1979) Electrophoretic transfer of proteins from polyacrylamide gels to nitrocellulose sheets: procedure and some applications. Proc Natl Acad Sci USA 76:4350-4354.

Trisler D, Schneider MD, Nirenberg M (1981) A topographic gradient of molecules in retina can be used to identify neuron position. Proc Natl Acad Sci USA 78:2145-2149.

Vermeersch CA, Stechmànn C, McLoon SC (1990) Comparison of immunosuppression techniques and their application to the production of monoclonal antibodies specific for axons on the nasal side of the developing retina. Soc Neurosci Abstr 16:319.

Walter J, Kern-Veits B, Huf J, Stolze B, Bonhoeffer F (1987a) Recognition of position-specific properties of tectal cell membranes by retinal axons in vitro. Development 101:685-696.

Walter J, Henke-Fahle S, Bonhoeffer F (1987b) Avoidance of posterior tectal membranes by temporal retinal axons. Development 101:909913. 